\title{
Denervação do Epicôndilo Lateral para o Tratamento da Epicondilite Lateral Crônica
}

Denervation of the Lateral Epicondyle for the Treatment of Chronic Lateral Epicondylitis

Denervación del Epicóndilo Lateral para el Tratamiento de la Epicondilitis Lateral Crónica

Luiz Henrique VILELA

Hospital Marcio Cunha, Fundação São Francisco Xavier, Ipatinga - MG, Brasil Henrique Gontijo CHAMON Hospital Marcio Cunha, Fundação São Francisco Xavier, Ipatinga - MG, Brasil Bruno Moraes VASCONCELOS Hospital Marcio Cunha, Fundação São Francisco Xavier, Ipatinga - MG, Brasil Luis Guilherme Rosifini Alves REZENDE Hospital das Clínicas da Faculdade de Medicina de Ribeirão Preto da Universidade de São Paulo, Ribeirão Preto - SP, Brasil https://orcid.org/0000-0002-2037-0135

Tiago Fernandes HERINGER Hospital Marcio Cunha, Fundação São Francisco Xavier, Ipatinga - MG, Brasil Fabricio Timo Abu KAMEL Hospital Marcio Cunha, Fundação São Francisco Xavier, Ipatinga - MG, Brasil Leandro Sampaio de FARIA

\section{Resumo}

Hospital Marcio Cunha, Fundação São Francisco Xavier, Ipatinga - MG, Brasil

Introdução: a epicondilite lateral é um processo crônico-degenerativo que acomete a origem dos músculos extensores do punho e dos dedos. Seu tratamento é realizado de forma conservadora, atingindo bons resultados. Porém, alguns casos evoluem de forma desfavorável, necessitando de intervenção cirúrgica. A denervação cirúrgica é uma das alternativas de tratamento, já descrita na literatura. Porém, poucos estudos sobre esta técnica estão disponíveis no contexto nacional. Objetivo: avaliar o resultado da denervação do epicôndilo lateral no tratamento da epicodilite lateral crônica. Métodos: estudo retrospectivo analisando 12 pacientes submetidos a denervação do epicôndilo lateral. O teste pré-operatório incluiu a injeção anestésica como preditor dos resultados. Os pacientes foram submetidos a denervação por neurectomia do(s) ramo(s) posterior(es) do nervo cutâneo posterior do antebraço através de incisão lateral no terço distal do braço. Avaliação clínica pré e pós-operatória dos pacientes foi realizada pelo questionário Quick DASH e EVA. Resultados: O resultado do Quick DASH foi de 78,7 pontos na avaliação pré-operatória e de 28,9 na avaliação pós-operatória $(p=0,001)$. O resultado da EVA pré-operatória foi de 7,58 pontos e 4,2 pontos na avaliação pós-operatória $(p=0,02)$. Conclusões: Concluímos que a técnica de denervação do epicôndilo lateral para o tratamento da epicondilite lateral crônica apresentou resultados satisfatórios. Porém, mais estudos são necessários para melhor avaliação da técnica.

Descritores: Articulação do Cotovelo; Cotovelo de Tenista; Denervação.

\section{Abstract}

Introduction: lateral epicondylitis is a chronic-degenerative process that affects the origin of the extensor muscles of the wrist and fingers. Its treatment is carried out conservatively, achieving good results. However, some cases evolve unfavorably, requiring surgical intervention. Surgical denervation is one of the management options already described in the literature. However, few studies on this technique are available in the national context. Aim of the study: To evaluate the denervation in the management of chronic lateral epicondylitis. Methods: retrospective study analyzing 12 patients undergoing lateral epicondyle denervation. Preoperative testing included anesthetic injection as a predictor of results. The patients underwent denervation of the posterior branch(s) of the posterior cutaneous nerve of the forearm through a lateral incision in the distal third of the arm. Pre and postoperative clinical assessment of patients was performed using the Quick DASH and VAS questionnaire. Results: The Quick DASH result was 78.7 points in the preoperative evaluation and 28.9 in the postoperative evaluation $(p=0.001)$. The preoperative VAS result was 7.58 points and 4.2 points in the postoperative evaluation ( $p=0.02)$. Conclusions: We conclude that the lateral epicondyle denervation technique has satisfactory outcomes. However, more studies are needed to evaluate the technique better.

Descriptors: Elbow Joint; Tennis Elbow; Denervation.

\section{Resumen}

Introducción: la epicondilitis lateral es un proceso crónico-degenerativo que afecta el origen de los músculos extensores de la muñeca y los dedos. Su tratamiento se realiza de forma conservadora, consiguiendo buenos resultados. Sin embargo, algunos casos evolucionan desfavorablemente, requiriendo intervención quirúrgica. La denervación quirúrgica es una de las alternativas de tratamiento, ya descrita en la literatura. Sin embargo, existen pocos estudios sobre esta técnica en el contexto nacional. Objetivo: evaluar el resultado de la denervación del epicóndilo lateral en el tratamiento de la epicodilitis lateral crónica. Métodos: estudio retrospectivo que analiza 12 pacientes sometidos a denervación del epicóndilo lateral. Las pruebas preoperatorias incluyeron inyección anestésicas como predictor de resultados. Los pacientes fueron sometidos a denervación mediante neurectomía de la (s) rama (s) posterior (s) del nervio cutáneo posterior del antebrazo a través de una incisión lateral en el tercio distal del brazo. La evaluación clínica pre y postoperatoria de los pacientes se realizó mediante el cuestionario Quick DASH y EVA. Resultados: El resultado Quick DASH fue de 78,7 puntos en la evaluación preoperatoria y 28,9 en la posoperatoria $(p=0,001)$. El resultado de la EVA preoperatoria fue de 7,58 puntos y de 4,2 puntos en la evaluación postoperatoria $(p=0,02)$. Conclusiones: Concluimos que la técnica de denervación del epicóndilo lateral para el tratamiento de la epicondilitis lateral crónica ha mostrado resultados satisfactorios. Sin embargo, se necesitan más estudios para evaluar mejor la técnica.

Descriptores: Articulación del Codo; Codo de Tenista; Desnervación. INTRODUÇÃO

A epicondilite lateral do cotovelo é uma patologia frequente, acometendo cerca de 1 a $3 \%$ da população, sendo mais comum na população não relacionada a práticas desportivas, quando comparada a prática de esportes. 'É comum ser denominada "cotovelo do tenista" ("tennis elbow"), apesar de estar presente em apenas 5 a $10 \%$ dos praticantes deste esporte. ${ }^{1,2}$ É uma patologia degenerativa (não inflamatória) que acomete preferencialmente a origem do músculo extensor radial curto do carpo. 
Histopatologicamente, observa-se um processo denominado degeneração angiofibroblastica ${ }^{2,3}$.

O tratamento é predominantemente conservador, realizado de forma longitudinal, ao longo de pelo menos um ano. Neste período diversos métodos como fisioterapia, medicamentos (analgésicos e AINES), infiltrações com corticosteroides ou outras substâncias podem ser utilizados ${ }^{4-9}$.

Não existe consenso em relação ao tratamento cirúrgico, principalmente referente ao tempo de sintomas ou gravidade da doença. De forma geral, alguns autores recomendam sua indicação após falha do tratamento conservador por 9 a 12 meses ${ }^{1,10,11}$. Além disso, não há consenso sobre a melhor técnica cirúrgica a ser utilizada. As alternativas cirúrgicas variam de tratamento convencional ("aberto"), percutâneo, minimamente-invasivo ou artroscópico. Apesar das diferentes técnicas, o objetivo cirúrgico é ressecar o tecido degenerado, podendo ou não associar a reinserção tendínea.

Apesar destes tratamentos expressarem bons resultados clínicos, não estão isentos de complicações. Também podem possuir custo elevado, tempo de recuperação prolongado, prorrogando o retorno às atividades ${ }^{1,12-16}$.

Existem alternativas como a denervação do epicôndilo lateral, que podem ser utilizadas, e apresentam bons resultados comparadas a outras técnicas, como desbridamento convencional ou artroscópico. A mesma pode ser realizada com anestesia local com ou sem sedação e de forma ambulatorial ${ }^{17-18}$.

O objetivo deste estudo, desenvolvido no Hospital Marcio Cunha - Fundação São Francisco Xavier, Ipatinga-MG, Brasil, é analisar os resultados do tratamento cirúrgico através da técnica de denervação do epicôndilo lateral do cotovelo em pacientes com epicondilite lateral crônica refratária ao tratamento conservador.

MATERIAL E MÉTODO

Estudo retrospectivo avaliando 17 pacientes submetidos ao tratamento cirúrgico através da técnica de denervação do epicôndilo lateral, operados entre 2013 a 2016. Os critérios de inclusão foram epicondilite lateral crônica, refratários ao tratamento conservador, submetidos ao tratamento cirúrgico com a técnica de denervação do epicôndilo lateral. Os critérios de exclusão foram pacientes que não compareceram aos retornos ambulatoriais. Ao final, 12 pacientes foram elegíveis para este estudo, sendo 8 pacientes do sexo feminino $(66,7 \%)$ e 4 do sexo masculino (33,3\%). Apenas um paciente estava afastado de suas atividades laborais.

\section{- Avaliação Pré-operatória}

Todos os pacientes foram submetidos a avaliação pré-operatória pela Escala Visual Análoga da Dor e pelo questionário QuickDASH, realizados 30 dias antes do procedimento cirúrgico. Os pacientes foram avaliados para a presença de dor nos testes de Mill e Cozen, além de dor no ponto do epicôndilo lateral $(2 \mathrm{~cm}$ anterior e distal ao mesmo).

\section{- Teste anestésico pré-operatório}

Todos os pacientes foram submetidos ao bloqueio anestésico. É realizada a delimitação anatômica do Ramo Posterior do Nervo Cutâneo Posterior do Antebraço (RP-NCPA), 4 centímetros proximal ao epicôndilo lateral, numa linha entre o epicôndilo lateral e a inserção do deltoide, onde realiza-se o bloqueio anestésico do nervo 30 dias antes do procedimento cirúrgico, com infiltração de $5 \mathrm{ml}$ de Xilocaína $2 \%$. Após, repetiu-se a avaliação dos testes de Mill e Cozen e da palpação do ponto do epicôndilo lateral, observando-se ausência de dor, sendo os mesmos elegíveis para o procedimento cirúrgico.

\section{- Avaliação Pós-operatória}

Todos os pacientes foram submetidos a avaliação pré-operatória pela Escala Visual Análoga da Dor e pelo questionário Quick$\mathrm{DASH}$, realizados 90 dias após o procedimento cirúrgico. Repetiu-se a avaliação dos testes de Mill e Cozen e da palpação do ponto do epicôndilo lateral e os seus resultados serão apresentados a seguir.

\section{- Técnica cirúrgica}

O paciente é posicionado em decúbito dorsal horizontal com presença de mesa de mão. É realizada assepsia e antissepsia com colocação dos campos estéreis. O membro é colocado em abdução de $30^{\circ}$ e em rotação interna com o antebraço pronado sobre a região do abdômen. Realiza-se a delimitação anatômica do Ramo Posterior do Nervo Cutâneo Posterior do Antebraço (RP-NCPA), numa linha entre $o$ epicôndilo lateral $e$ a inserção do deltoide, onde realiza-se o bloqueio anestésico do nervo. A cirurgia é realizada com uso de torniquete. Uma incisão de $5 \mathrm{~cm}$ é realizada ao longo do trajeto do RP-NCPA, 3 a $8 \mathrm{~cm}$ proximal ao epicôndilo lateral, na região lateral do braço (Figura 1). Realiza-se dissecção romba, observando o nervo geralmente na porção subcutânea ou subfascial (Figura 2) ${ }^{17}$.

Após identificar o nervo, ele é dissecado em direção distal em busca dos seus ramos epicondilares (1 a 3 ramos). Estes ramos direcionam-se para o Epicôndilo Lateral. O nervo cutâneo posterior do antebraço continua 
em direção distal para inervar o antebraço, de forma longitudinal e tem maior calibre. Realizase tração nos ramos para o epicôndilo observando-se uma mobilização na pele sobre este. Estes ramos são dissecados, mobilizados e a neurectomia é realizada, inserindo os cotos dentro da fáscia, evitando a formação de neuroma (Figura 3).

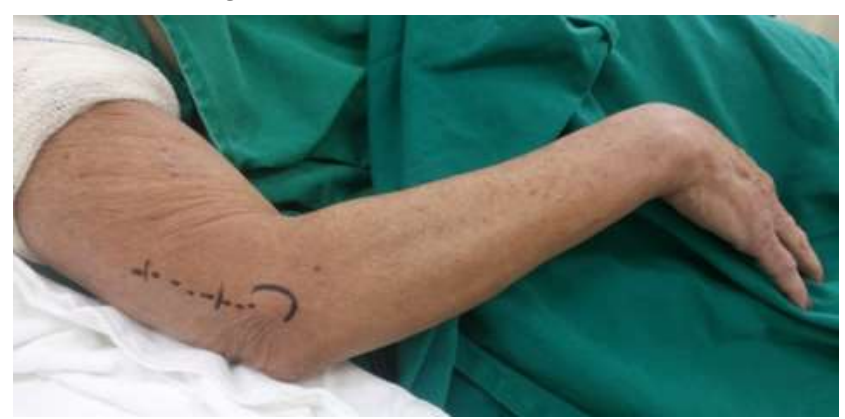

Figura 1: Landmarks do Epicondilo Lateral e via de acesso

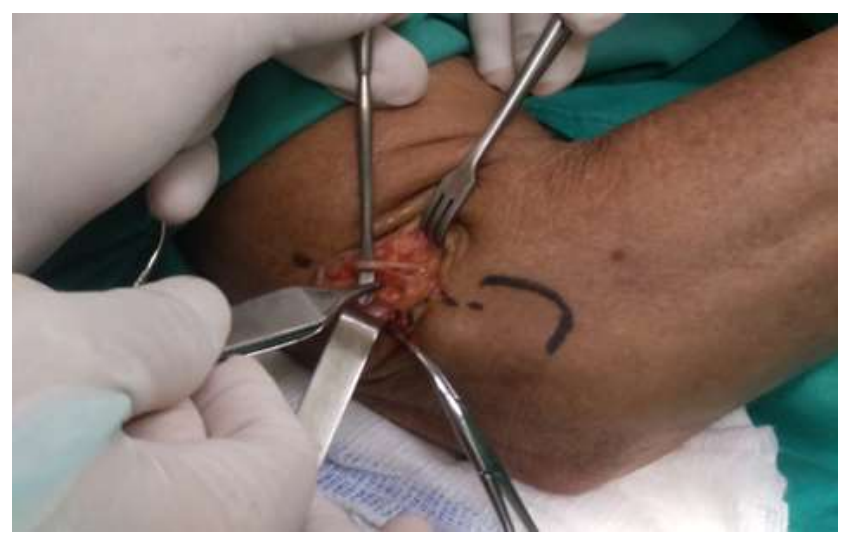

Figura 2: Identificação do nervo cutâneo posterior do antebraço.

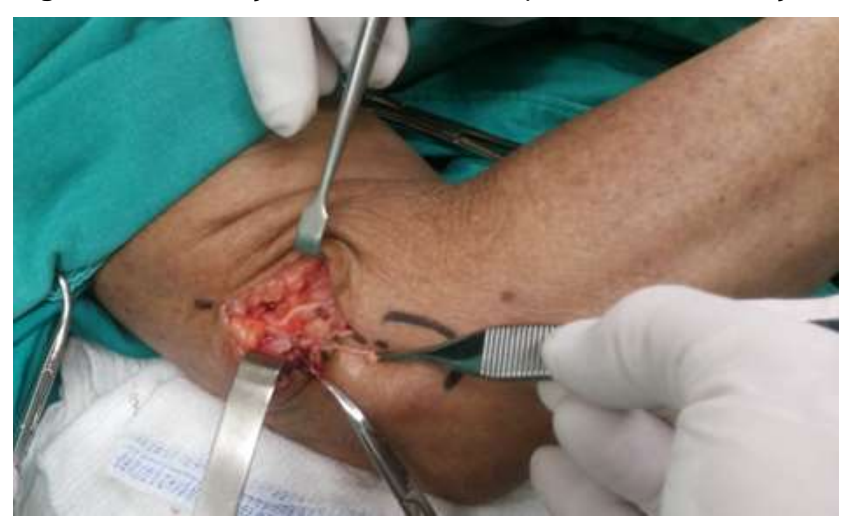

Figura 3: Neurectomia do ramo posterior do cutâneo posterior do antebraço.

Após, retira-se o torniquete para boa hemostasia e sutura da pele com fios absorvíveis e curativo oclusivo. Durante o pósoperatório o paciente é liberado no pósoperatório imediato para realizar amplitude de movimento e atividades conforme tolerância, incluindo esporte.

RESULTADOS

De forma absoluta, $75 \%$ dos pacientes apresentaram graus reduzidos e/ou pouco significativos de limitações para realização das tarefas (diárias e de trabalho) ou dor, sendo que apenas 3 pacientes registraram graus mais significativos. A média na pontuação EVA pósoperatória foi de 7,58 pontos (variando de 6 a 9), comparada ao valor de 4,2 pontos (variando de 1 a 8) no pós-operatório. Observamos uma diferença estatisticamente significativa $(p=0.002)$ com base no teste $T$ de Student. Os dados encontram-se disponíveis na Tabela 1.

Tabela 1. Resultados EVA pré-operatória e pós-operatório. Valor de $p$ obtido com base no teste T de Student.

\begin{tabular}{l|c|c|c|c|c|c|c|c|c|c|c|c|c}
\hline Paciente & 1 & 2 & 3 & 4 & 5 & 6 & 7 & 8 & 9 & 10 & 11 & 12 & Média* $^{*}$ \\
\hline EVA & & & & & & & & & & & & & 7,58 \\
Pré-Operatória & 8 & 9 & 6 & 8 & 6 & 6 & 7 & 8 & 8 & 8 & 9 & 8 & 6 a 9 \\
\hline $\begin{array}{l}\text { EVA } \\
\text { Pós-Operatório }\end{array}$ & 2 & 8 & 6 & 1 & 2 & 5 & 8 & 5 & 5 & 6 & 2 & 1 & $\begin{array}{c}4,2 \\
1 \text { a } 8\end{array}$ \\
\hline $\begin{array}{l}\text { Valor de } p=0,02 \\
\text { *Média (Variância) }\end{array}$
\end{tabular}

Observamos uma média de pontuação do Quick-DASH média pré-operatória de 78,7 (variando de 60 a 91,7). Já no período pósoperatório, observamos uma pontuação de 28,9 (variando de 5 a 86.7) no Quick-DASH. Observamos uma diferença estatisticamente significativa $(p<0.001)$ com base no teste $T$ de Student. Além disso, a menor pontuação registrada no pós-operatório foi 5 (P6 e P9) e maior foi 86.7 pontos para a paciente P2. Os dados encontram-se disponíveis na Tabela 2.

Tabela 2. Resultados Quick-DASH pré-operatória e pósoperatório. *Valor de p obtido com base no teste T de Student.

\begin{tabular}{|c|c|c|c|c|c|c|c|c|c|c|c|c|c|}
\hline Paciente & 1 & 2 & 3 & 4 & 5 & 6 & 7 & 8 & 9 & 10 & 11 & 12 & Média* \\
\hline $\begin{array}{l}\text { Quick-DASH } \\
\text { Pré-Operatória }\end{array}$ & 81,7 & 90 & 61,7 & 83,3 & 66,7 & 60 & 75 & 81,7 & 86,7 & 85 & 91,7 & 81,7 & \begin{tabular}{|l|}
78,7 \\
$(60$ a 91,7$)$ \\
\end{tabular} \\
\hline $\begin{array}{l}\text { Quick-DASH } \\
\text { Pós-Operatório }\end{array}$ & 23,3 & 86,7 & 6,7 & 18,3 & 26,7 & 5 & 8,3 & 56,7 & 5 & 68,3 & 26,7 & 15,0 & $\begin{array}{l}28,9 \\
(6 \text { a } 86,7)\end{array}$ \\
\hline
\end{tabular}

DISCUSSÃO

O tratamento da epicondilite lateral ainda é controverso, seja quando optado pelo tratamento conservador ou cirúrgico. Muitos autores evitam o tratamento cirúrgico devido a possibilidade de não melhora e litígio no contexto trabalhista. Espera-se uma melhora do quadro clínico com um tratamento conservador após 9 a 12 meses, quando realizado de forma adequada. Contudo, não existe padronização para o tratamento conservador ou melhores protocolos, o que é motivo de discussão entre diversos autores. ${ }^{8,9,19-21}$ Smidt et al. $^{18}$ observaram que a infiltração com corticoides parece ser o tratamento mais eficaz até 6 meses, porém, tanto a fisioterapia quanto o tratamento expectante (observação sem intervenção) eram eficazes em 12 meses.

Já em relação ao tratamento cirúrgico, a revisão sistemática Pierce et al. não demostrou diferenças significativas de melhora em relação ao tratamento aberto (convencional) ou artroscópico; ainda resultados inferiores para o método percutâneo ${ }^{13}$. A modalidade percutânea apresenta vantagens relativas ao baixo custo e rapidez de retorno as funções, porém, com 
taxas de sucesso variáveis, como observado por outros autores ${ }^{19}$.

A denervação do cotovelo é um método terapêutico introduzido por Kaplan ${ }^{20}$ em 1959, baseado em estudos anatômicos de Gardner $^{8}$ em 1948. Mais tarde, Wilhelm publicou um estudo observacional em 166 pacientes, apresentando uma taxa de $90 \%$ de sucesso ${ }^{9,21}$. Sua técnica associava a desinserção tendínea do supinador, ancôneo, tríceps e extensores radiais) ao processo de denervação, associada também a descompressão do nervo radial ou interósseoposterior $^{9,21}$.

Recentemente, Berry et al. $^{17}$ compararam a epicondilectomia isolada, a epicondilectomiia associada a denervação e a denervação isolada. A denervação isolada apresentou o melhor resultado em relação a melhora dos sintomas e maior rapidez de retorno as atividades. Mais tarde Rose et al. ${ }^{16}$, em 2013 publicaram o último artigo contendo detalhes técnicos com uma casuística maior e $80 \%$ de bons resultados.

Existem poucos estudos realizados ao nível nacional, envolvendo esta técnica de denervação, apresentando resultados palpáveis para a realidade nacional. Apesar de uma casuística pequena, obtivemos bom resultado pós-operatório, com nível de evidência satisfatório. Apesar das taxas de procedimento cirúrgico para a epicondilite lateral serem baixas, este estudo é capaz de contribuir com uma modalidade cirúrgica, em língua portuguesa, como alternativa técnica em casos de epicondilite lateral crônica, refratárias ao tratamento conservador.

Além disto, a simplicidade deste procedimento permite a realização desta técnica de forma ambulatorial, permitindo rápido retorno funcional, quando comparada à epicondilectomia ou liberação ou desbridamento da origem dos extensores.

Acreditamos que 0 tratamento conservador é a melhor modalidade terapêutica, tendo em vista, que a epicondilite lateral é extremamente comum no cotidiano ortopédico, com alta taxa de sucesso. Porém, a denervação pode ser uma alternativa viável em casos crônicos, superiores a 12 meses e refratários, onde houve esgotamento das alternativas nãocirúrgicas.

CONSIDERAÇÕES FINAIS

Concluímos que a técnica de denervação do epicôndilo lateral para o tratamento da epicondilite lateral crônica apresentou resultados satisfatórios. Porém, mais estudos com uma amostra maior são necessários, bem como estudos com grupo controle para comparar a eficácia desta técnica comparada às demais modalidades terapêuticas.

REFERÊNCIAS

1. Cohen M, Filho GRM. Epicondilite lateral do cotovelo. Rev Bras Ortop. 2012;47(4):414-20

2. Lech O, Piluski PCF, Severo AL. Epicondilite lateral do cotovelo. Rev Bras Ortop, 2003;38(8):421-36.

3. Nirschl RP. Elbow tendinosis / tennis elbow. Clin Sports Med. 1992;11(4):851-70

4. Nirschl RP. Muscle and tendon trauma: tennis elbow tendinosis in: Morrey B F, The Elbow and its disorders, 3a ed. Philadelphia, Saunders, 2000, 523-535.

5. Dojode CM. A randomised control trial to evaluate the efficacy of autologous blood injection versus local corticosteroid injection for treatment of lateral epicondylitis. Bone Joint Res. 2012;1(8):192-97.

6. Kertzman P, Lenza M, Pedrinelli A, Ejnisman B. Tratamento por ondas de choque nas doenças musculoesqueléticas e consolidação óssea Análise qualitativa da literatura. Rev Bras Ortop. 2015;50(1):3-8.

7. Wong SM, Hui AC, Tong PY et al. Treatment of lateral epicondylitis with botulinum toxin: a randomized, double-blind, placebo-controlled trial. Ann Intern Med. 2005;143(11):793-97

8. Gardner E. The innervation of the elbow joint. Anat Rec. 1948;102(2):161-74.

9. Wilhelm A. Die Behandlung der Epicondylitis humeri radialis durch Dekompression des $\mathrm{N}$. radialis [Management of epicondylitis humeri radialis through the decompression of the radial nerve]. Handchirurgie. 1977;9(4):185-7.

10. Hayton MJ, Santini AJ, Hughes PJ, Frostick SP, Trail IA, Stanley JK. Botulinum toxin injection in the treatment of tennis elbow. A double-blind, randomized, controlled, pilot study. J Bone Joint Surg Am. 2005;87(3):503-7.

11. Medina GEIS, Roselis DG, Sano $M$ et al. Tratamento da epicondilite lateral do cotovelo por mini incisão. Rev Bras Ortop lustr. $2011 ; 2(2): 43-52$.

12. Miazaki A, Fregonese $M$, Santos PD et al. Avaliação dos resultados do tratamento artroscópico da epicondilite lateral. Rev Bras Ortop. 2010;45(2):136-40.

13. Pierce TP, Issa K, Gilbert BT et al. A Systematic Review of Tennis Elbow Surgery: Open Versus Arthroscopic Versus Percutaneous Release of the Common Extensor Origin. Arthroscopy. 2017;33(6): 1260-68.

14. Solheim E, Hegna J, Øyen J. Arthroscopic versus open tennis elbow release: 3 - to 6-year results of a case-control series of 305 elbows. Arthroscopy. 2013;29(5):854-59 
15. Carofino BC, Bishop AT, Spinner RJ, Shin AY. Nerve injuries resulting from arthroscopic treatment of lateral epicondylitis: report of 2 cases. J Hand Surg Am. 2012;37(6):1208-10.

16. Rose NE, Forman SK, Dellon AL. Denervation of the lateral humeral epicondyle for treatment of chronic lateral epicondylitis. J Hand Surg Am. 2013;38(2):344-49.

17. Berry N, Neumeister MW, Russell RC, Dellon AL. Epicondylectomy versus denervation for lateral humeral epicondylitis. Hand $(\mathrm{N} Y)$. 2011;6(2):174-78.

18. Smidt N, van der Windt DA, Assendelft WJ et al. Corticosteroid injections, physiotherapy, or a wait-and-see policy for lateral epicondylitis: a randomised controlled trial. Lancet. 2002;359(9307):657-62

19. Mattie R, Wong J, McCormick Z, Yu S, Saltychev M, Laimi K. Percutaneous Needle Tenotomy for the Treatment of Lateral Epicondylitis: A Systematic Review of the Literature. PM R. 2017;9(6):603-11.

20. Kaplan EB. Treatment of tennis elbow (epicondylitis) by denervation. J Bone Joint Surg Am. 1959;41-A(1):147-51.

21. Wilhelm A. Tennis elbow: treatment of resistant cases by denervation. J Hand Surg $\mathrm{Br}$. 1996;21(4):523-33.

\section{CONFLITO DE INTERESSES}

Os autores declaram não haver conflitos de interesse

\section{AUTOR PARA CORRESPONDÊNCIA}

\section{Luiz Henrique Vilela \\ Hospital Marcio Cunha, \\ Fundação São Francisco Xavier \\ Rua México, №30 - Bairro Cariru, 35160-123 Ipatinga - MG, Brasil \\ e-mail: Ihvilela41@gmail.com}

Submetido em 18/10/2021

Aceito em 04/01/2022 